\title{
SOME REMARKS ON CONFORMAL PSEUDO-RIEMANNIAN ACTIONS OF SIMPLE LIE GROUPS
}

\author{
Charles Frances and Abdelghani ZeghiB
}

ABSTRACT. We improve recent results on conformal pseudo-Riemannian actions of simple Lie groups obtained by U. Bader and A. Nevo, and get optimal statements.

\section{Introduction}

A general motivation of our work is to understand actions of "big" Lie groups preserving a rigid geometric structure on a compact manifold. An account on this subject can be found, for instance, in [4], where it is vaguely conjectured that such actions should be sufficiently peculiar to be "classified". Yet, many constructions show that this is by no means a simple matter, and even very special situations still resist to existing approaches. In a recent work [1], U. Bader and A. Nevo made a substantial progress towards the understanding of conformal actions of simple Lie groups on compact pseudo-Riemannian manifolds. The technics they used relied essentially on Lie algebraic considerations, in a way that was pioneered a few years ago by N. Kowalsky (see [10]). Our aim in these "remarks" is to show how extra geometrical and dynamical arguments allow us to sharpen these results, in order to get optimal statements.

To fix the notations, let $\mathbb{R}^{p, q}$ be the Minkowski space of type $(p, q)$, i.e. $\mathbb{R}^{p+q}$ endowed with the quadratic form of type $(p, q):-d x_{1}^{2}-\ldots-d x_{p}^{2}+d y_{1}^{2}+\ldots+d y_{q}^{2}$.

One defines a pseudo-Riemannnian manifold $M$ of type $(p, q)$ as a manifold such that the tangent space at each point is endowed with a non degenerate quadratic form of signature $(p, q)$ (the distribution of these quadratic forms being moreover smooth). Given a pseudo-Riemannian metric $g_{0}$ of signature $(p, q)$ on $M$, the conformal structure associated to $g_{0}$ is the class of metrics which are conformal to $g_{0}$, i.e. of the form $e^{\sigma} g_{0}$, for some smooth function $\sigma$. Equivalently, two pseudo-Riemannian metrics are conformal if and only if they determine the same isotropic cone (of course, we exclude here the "trivial" Riemannian case).

A distinguished class of conformal pseudo-Riemannian manifolds is the locally conformally flat ones, i.e. those which are locally conformal to the Minkowski space $\mathbb{R}^{p, q}$. An important example of such a manifold is the conformal compactification of Minkowski space, denoted by $C^{p, q}$. This later space can be defined as the projection of the isotropic cone of the space $\mathbb{R}^{p+1, q+1}$ on the projective space $\mathbb{R} \mathbb{P}^{p+q+1}$. The manifold $C^{p, q}$ inherits from $\mathbb{R}^{p+1, q+1}$ a natural conformal

Received April 24, 2004. 
structure of type $(p, q)$, which is conformally flat and invariant under the action of $O(p+1, q+1)$. In fact, $C^{p, q}$ admits a conformal two-fold covering, which is the product $\mathbb{S}^{p} \times \mathbb{S}^{q}$ endowed with the conformal class of $-g_{\mathbb{S}^{p}}+g_{\mathbb{S}^{q}}$. Let us recall two fundamental properties of the space $C^{p, q}$. First of all, the existence of a stereographic projection, which embeds conformally the space $\mathbb{R}^{p, q}$ as a dense open subset of $C^{p, q}$. Secondly, a theorem of Liouville on $C^{p, q}$, asserting that when $p+q \geq 3$, any conformal diffeomorphism between open subsets of $C^{p, q}$ extends to a unique element of $O(p+1, q+1)$ (see for instance [12] or [6]). The two previous results show that $C^{p, q}$ is the universal substratum of conformally flat pseudo-Riemannian geometry of type $(p, q)$. Indeed, in dimension $\geq 3$, a conformal pseudo-Riemannian manifold of type $(p, q)$ is conformally flat if and only if it supports a $\left(O(p+1, q+1), C^{p, q}\right)$-structure.

We will always consider in the following, manifolds of dimension $\geq 3$. Moreover, to simplify notations, we adopt the convention $p \leq q$. Since we are not concerned with the Riemannian case, we also assume $1 \leq p$. Of course, the translation of our statements to the case where $p \geq q \geq 1$ is straightforward.

In [1], the authors consider a simple Lie group $G$ with finite center, acting conformally on a compact pseudo-Riemannian manifold of signature $(p, q)$. They first prove that the real rank of $G$, denoted by $r k_{\mathbb{R}}(G)$, must satisfy the inequality $r k_{\mathbb{R}}(G) \leq p+1$ (recall that for us, $p \leq q$ ). Of course, one wonders what can be said when the rank of $G$ is the maximal possible. The main result of [1] answers partially this question:

Theorem 1. [1] Let $G$ be a connected simple Lie group with finite center acting smoothly and conformally on a smooth compact pseudo-Riemannian manifold $M$ of type $(p, q)$. Assume the (real) rank of $G$ equals $p+1$. Then:

- The group $G$ is locally isomorphic to $S O^{o}(p+1, k+1)$, for some $k$ such that $p \leq k \leq q$.

- There exists a closed $G$-orbit, which is conformally equivalent to a finite cover of $C^{p, k}$.

- In particular, if the $G$ action is minimal, then $M$ is conformally equivalent to a finite cover of $C^{p, k}$.

It is the last part of the statement, which suggests that a rigidity property should hold for these actions. Unfortunately, it involves a minimality extracondition. Our main result is to relax this condition (by the way, we will also relax the finiteness condition on the center of the Lie group). In the non-Lorentzian case, i.e. the case $p \geq 2$, we get the

Theorem 2. Let $G$ be a connected simple Lie group acting smoothly and conformally on a smooth compact pseudo-Riemannian manifold $M$ of type $(p, q)$ with $p \geq 2$. If the rank of $G$ equals $p+1$, then:

- The group $G$ is locally isomorphic to $S O^{\circ}(p+1, k+1)$ for some $k$ such that $p \leq k \leq q$.

- Up to finite cover, $M$ is conformally equivalent to the space $C^{p, k}$. 
Let us precise here that "up to finite cover" means, for us, "after a finite number of manipulations consisting in taking finite quotients or finite covers".

In the Lorentzian case, i.e. when $p=1, M$ is not necessarily $C^{1, k}$, but all possibilities are completely described as follows:

Theorem 3. Let $G$ be a connected simple Lie group of rank 2, acting smoothly and conformally on a smooth compact Lorentz manifold $M$ of dimension $n$. Then:

- The group $G$ is locally isomorphic to $S O^{\circ}(2, k)$ for some $k$ such that $3 \leq$ $k \leq n$.

- $M$ is, up to finite cover, a complete conformally flat structure on $\mathbb{S}^{1} \times \mathbb{S}^{n-1}$, i.e $M$ is a quotient of $\widetilde{C^{1, n-1}}$ (the universal cover of $C^{1, n-1}$ ) by an infinite cyclic group $\Gamma$.

- The possible groups $\Gamma$ are those generated by any element in a product $\mathbb{Z}^{*} \times O(n-k) \subset \widehat{O^{o}(2, n)}$ (the universal cover of $O^{o}(2, n)$ ), where the $\mathbb{Z}$ factor is the center of $\widehat{O(2, n)}$.

Remark 4. In the last theorem, it follows easily from the proof that when $k=n$ or $k=n-1$, the manifold $M$ is, up to finite cover, conformally equivalent to the space $C^{1, n-1}$.

\section{Proofs of the main theorems}

2.1. About the finiteness of the center. We first study what become the conclusions of theorem 1 if we remove the asumption that the center of $G$ is finite.

Theorem 5. Let $G$ be a simple Lie group acting smoothly and conformally on a smooth compact pseudo-Riemannian manifold $M$ of type $(p, q)$. If the rank of $G$ equals $p+1$, then:

- The group $G$ is locally isomorphic to $S O^{\circ}(p+1, k+1)$ for $p \leq k \leq q$. In particular, its Lie algebra is the full Lie algebra of conformal vector fields of $\mathbb{R}^{p, q}$ (or, equivalently, of any open subset of $\mathbb{R}^{p, q}$ ).

- Each G-invariant closed subset contains a G-orbit $N$ which is locally conformally flat.

Proof. we have to recall the main steps of the proof of theorem 1 . The authors prove first the existence of a point $m \in M$ such that $\operatorname{Stab}_{G}(m)=H$ is normalized by a real algebraic cocompact group $Q \subset G$. It is done thanks to the remark that the points of $M$ where the orbits have minimal dimension $l$ is a compact subset $M^{\prime} \subset M$, and the existence of a $G$-equivariant continuous Gauss map $\psi: M^{\prime} \longrightarrow \mathbb{P}\left(\mathfrak{g}^{\star} \otimes \mathfrak{g}^{\star}\right) \times G r_{l}(\mathfrak{g})$ (where $G r_{l}(\mathfrak{g})$ denotes the Grassmannian of subspaces of dimension $l$ in $\mathfrak{g})$. The conclusion is then obtained by looking at the algebraic action of $G$ on $\mathbb{P}\left(\mathfrak{g}^{\star} \otimes \mathfrak{g}^{\star}\right) \times G r(\mathfrak{g})$ (section 4 of [1]). If we consider a closed $G$-invariant subset $F$ instead of $M$ itself, the points of $F$ the orbits of 
which have minimal dimension are still a $G$-invariant compact subset, and one can still define the map $\psi$. So, also in this case, a point $m$ as above exists. The existence of such a point, and a case by case study involving Lie algebras lead the authors to prove that $\mathfrak{g}$ has to be isomorphic to $\mathfrak{s o}(p+1, k+1)$. Remark that this Lie algebra is exactly the Lie algebra of conformal vector fields of $\mathbb{R}^{p, q}$, by Liouville's theorem. On the other hand, $\mathfrak{h}$ is isomorphic to the Lie algebra of the parabolic subgroup $P$, stabilizer of a point in $C^{p, k}$, for the action of $O(p+1, k+1)$ (see [1] section 5). Until this point of the proof, no asumption on the center of $G$ is needed. Now, making the asumption that $G$ has a finite center, the authors get that $G / P$ is compact, and the orbit of $m$ as well. Moreover, since the action of $A d(H)$ on $\mathfrak{g} / \mathfrak{h}$ can leave invariant a single pseudo-Riemannian conformal class, the orbit has to be (up to finite cover) conformally equivalent to $C^{p, k}$. Now, without the finiteness asumption on the center, the orbit $N$ of $m$ may not be closed, but remains locally conformally equivalent to $C^{p, k}$.

2.2. Conformal flatness. We can now prove:

Theorem 6. Let $G$ be a simple Lie group acting conformally on a compact pseudo-Riemannian manifold $M$ of type $(p, q)$, such that the rank of $G$ equals $p+1$. Then $M$ is conformally flat.

Proof. We consider a $G$-orbit $N$ given by theorem 5 . Let $x$ be a point of $N$, and $H=\operatorname{Stab}_{G}(m)$. There is a neighbourhood of $x$ in $N$ which is conformally equivalent to some neighbourhood $V$ of 0 in $\mathbb{R}^{p, q}$. The Lie algebra $\mathfrak{h}$ is exactly the Lie algebra of conformal vector fields of $V$ vanishing at 0 . So, in [articular, there is a subgroup $H^{\prime} \subset H$, isomorphic to $\mathbb{R} \times S O^{o}(p, k)$ (up to finite index), and acting faithfully on $T_{x} N$. This action is moreover the same as the linear action of $\mathbb{R} \times S O^{o}(p, k)$ on $\mathbb{R}^{p, k}$.

The action of $S O^{\circ}(p, k) \subset H^{\prime}$ on $T_{x} M$ is easy to describe. On $T_{x} N$, it is, as we just said, the usual linear action on $\mathbb{R}^{p, k}$. On $T_{x} N^{\perp}$, the action is trivial. Indeed $T_{x} N^{\perp}$ has Riemannian signature, and since $S O^{\circ}(p, k)$ is simple without compact factor, any morphism from $S O^{\circ}(p, k)$ in a compact group has to be trivial. Now, the following theorem ensures that in a neighbourhood of $x$, the action of $S O^{\circ}(p, k)$ is linearizable.

Theorem 7. If a semi-simple Lie group acts conformally on a pseudo-Riemannian manifold by fixing some point, then its action is linearizable near that point.

We will prove this result in $\S 3$.

Thanks to theorem 7 , we know that in some neighbourhood of $x$, the fixed points of $S O^{\circ}(p, k)$ constitute a smooth submanifold $N^{\perp} \subset M$. At $x$, one has $T_{x}\left(N^{\perp}\right)=\left(T_{x} N\right)^{\perp}$. The $\mathbb{R}$-action commutes with $S O^{\circ}(p, k)$, and hence it preserves $N^{\perp}$. (Actually, in order to get a global $N^{\perp}$ we saturate it by the $\mathbb{R}$-action).

Let us choose an element $f$ in $H^{\prime}$ lying in the $\mathbb{R}$ factor. The differential $D_{x} f$ acts as a homothety of distorsion $\lambda$ on $T_{x} N$. But since $N$ is of signature $(p, k)$ 
and $D_{x} f$ must be in $\mathbb{R} \times O(p, q)$ ( $f$ is a conformal transformation of $\left.M\right)$, the action of $D_{x} f$ on $T_{x} N^{\perp}$ is that of a euclidean similarity $\lambda R(R \in O(n))$.

The conformal Riemannian manifold $N^{\perp}$ has therefore an essential (i.e. non proper) conformal group. By Ferrand's Theorem [5] (in both the compact and the non compact case), this manifold is conformally a standard sphere or a Euclidean space. In any case, the action of $f$ on $N^{\perp}$ is smoothly linearizable near $x$, so it is smoothly locally conjugated to the action of $\lambda R$ in a neighbourhood of 0 . Moreover, on $\left(T N^{\perp}\right)^{\perp}$, the action of $D f$ has to commute with that of $O(p, k)$ (which is irreducible). Therefore, in some trivialisation $\mathbb{R}^{q-k} \times \mathbb{R}^{q-k} \times \mathbb{R}^{p, k}$ of $T M$ over a neighbourhood $U$ of $x$ in $N^{\perp}$, the action of $D f$ is as follows: $(y, u, v) \longmapsto(\lambda R . y, \lambda R . u, \lambda v)$.

Let us suppose that $f$ is a contraction, i.e that $0<\lambda<1$, and consider $W$ the Weyl tensor of the conformal structure [11]. This tensor vanishes if $M$ has dimension 3 , so we suppose in a first time that the dimension of $M$ is $\geq 4$. Let us put the euclidean norm $\mathcal{N}$ on the factor $\mathbb{R}^{q-k} \times \mathbb{R}^{p, k}$, we get that for $y \in U, v \in T_{y} M$ and $n \in \mathbb{N}, \mathcal{N}\left(D f_{y}^{n}(v)\right)=\lambda^{n} \mathcal{N}(v)$. Now let $X, Y, Z \in T_{y} M$, and set $T=W_{y}(X, Y, Z)$. By conformal invariance of the Weyl tensor, one gets $D f_{y}^{n} T=W_{f^{n}(y)}\left(D f^{n} X, D f^{n} Y\right) D f^{n} Z$. But the norm $\mathcal{N}\left(D f_{y}^{n} T\right)$ behaves like $\lambda^{n} \mathcal{N}(T)$ while the norm $\mathcal{N}\left(W_{f^{n}(y)}\left(D f^{n} X, D f^{n} Y\right) D f^{n} Z\right)$ behaves like $\lambda^{3 n} r_{n}$ (where $r_{n}$ is a bounded sequence of reals). We get a contradiction as $n$ tends to $+\infty$, unless $W=0$ at $y$, and hence all along $N^{\perp}$.

When $x$ runs over $N$, the corresponding submanifolds $N^{\perp}$, fills a neighborhood of $N$ in $M$. Therefore the Weyl tensor vanishes on an open $G$-invariant set $\Omega$.

To conclude, let us consider a maximal G-invariant open set $\Omega$ on which the Weyl tensor vanishes. If it is not the whole $M$, we apply theorem 5 to its complementary $F$. Doing the proof above once again, we will find some $x \in F$ such that the Weyl tensor vanishes on some neighbourhood of $x$. This yields a contradiction with the maximality of $\Omega$. We conclude that $W=0$ on the whole $M$, i.e $M$ is conformally flat.

When $M$ has dimension 3, another tensor (the Schouten tensor of type $(2,1)$ ) is left invariant by $G$, and vanishes if and only if the manifold is conformally flat. We prove by the same arguments as above that such a tensor must be identically zero.

2.3. Proof of Theorem 2. Let $M$ be as in Theorem 2. We infer from Theorem 6 that $M$ is conformally flat. Therefore, there is a holonomy morphism $\rho$ : $\pi_{1}(M) \longrightarrow O(p+1, q+1)$ and a developping map $\delta$ from the universal cover $\tilde{M}$ to $\widetilde{C^{p, q}}$, the universal cover of $C^{p, q}$. The map $\delta$ is a conformal local diffeomorphism, which is $\rho$-equivariant. The holonomy morphism $\rho$ extends to a morphism $\bar{\rho}$ : $\operatorname{Conf}(\tilde{M}) \longrightarrow O(p+1, q+1)$, and $\delta$ is also $\bar{\rho}$-equivariant. By theorem 5 , the group $G$ is locally isomorphic to $S O^{o}(p+1, k+1)$ for $p \leq k \leq q$. Now, since we are not in the Lorentzian case, the fundamental group of $S O^{\circ}(p+1, k+1)$ is finite and so, up to finite index, we can suppose that $G=S O^{\circ}(p+1, k+1)$. Then, $\bar{\rho}$ 
embeds the group $G$ in $O(p+1, q+1)$. But up to conjugacy, there is a unique way to embed $S O^{\circ}(p+1, k+1)$ in $O(p+1, q+1)$, and looking at the canonical one, we see that the centralizer of $\bar{\rho}(G)$ in $O(p+1, q+1)$ is a compact subgroup $K \subset O(p+1, q+1)$, which is isomorphic to $O(q-k)$. Since $G$ acts on $M$, and so is centralized by $\pi_{1}(M)$, the image of the holonomy homomorphism is contained in $K$. But up to conjugacy, $K$ is included in a product $O(p+1) \times O(q+1)$, so that our structure is in fact a $\left(\widetilde{C^{p, q}}, O(p+1) \times O(q+1)\right)$-structure. It is a standard fact (see e.g [15] proposition 3.4.10) that such a structure on a compact manifold has to be complete. In other words, $\delta$ is a diffeomorphism and $M$ is a finite quotient of $\widetilde{C^{p, q}}$ (which is itself a double covering of $C^{p, q}$ ).

2.4. Proof of Theorem 3. As in the previous discussion we get that $G$ is locally isomorphic to $S O^{\circ}(2, k)$ for $3 \leq k \leq n$. Let us consider the developping $\operatorname{map} \delta: \tilde{M} \longrightarrow \widetilde{C^{1, n-1}}$ and the holonomy morphism $\rho: \pi_{1}(M) \longrightarrow \widetilde{O(2, n)}$. We denote by $\pi$ the projection from $\widetilde{C^{1, n-1}}$ to $C^{1, n-1}$. We call $\hat{\delta}$ the composition $\pi \circ \hat{\rho}$ and $\hat{\rho}$ the morphism $\rho$ followed by the projection on $O(2, n)$. Since $G$ is locally isomorphic to $S O^{\circ}(2, k)$, there is a family of conformal vector fields on $M$ whose Lie algebra is $\mathfrak{s o}(2, k)$. Now, looking at the image of these vector fields by $\hat{\delta}$, we get again that the image of the morphism $\hat{\rho}$ centralizes a subgroup of $O(2, n)$ which is isomorphic to $O(2, k)$. As in the previous proof, we deduce that the image $\hat{\rho}\left(\pi_{1}(M)\right)$ is included in some compact group $K$ isomorphic to $O(n-k)$. Now, the lift of any compact subgroup of $O(2, n)$ is included, up to conjugacy, in a product $\mathbb{R} \times O(n) \subset \widetilde{O(2, n)}$. Recall that $\widetilde{C^{1, n-1}}$ is $\mathbb{R} \times \mathbb{S}^{n-1}$. The $\mathbb{R}$-factor of $\mathbb{R} \times O(n)$ acts by translation on $\mathbb{R}$ and trivially on $\mathbb{S}^{n-1}$, and the $O(n)$ factor acts trivially on $\mathbb{R}$ and by rotations on $\mathbb{S}^{n-1}$. Observe that $\mathbb{R} \times O(n)$ acts transitively on $\widetilde{C^{1, n-1}}$ with compact stabilizers. Then, proposition 3.4 .10 of [15] ensures that the structure on $M$ is complete, i.e $\delta$ is a diffeomorphism. Now, observe that any discrete subgroup of $\mathbb{R} \times O(n)$ yielding a compact quotient of $\widetilde{C^{1, n-1}}$ is infinite cyclic (up to finite index). Moreover, quotients by such infinite cyclic groups are always finitely covered by $\mathbb{S}^{1} \times \mathbb{S}^{n-1}$. On the other hand, an element $(t, \sigma) \subset \mathbb{R} \times O(n)$ centralizes a subgroup with Lie algebra $\mathfrak{s} o(2, k)$ exactly when $t=k \pi, k \in \mathbb{Z}^{*}$ (recall that $(\pi, I d)$ generates the center of $\left.\widehat{O(2, n)}\right)$, and $\sigma$ is included in some subgroup $O(n-k) \subset O(n)$.

\section{Proof of Theorem 7}

Much has been written about (local) linearization of a semi-simple Lie group action near a fixed point (see [2] for a report on the question). Essentially, it is known that linearization is possible in the analytic case, but not always in the smooth one. Theorem 7 is a first evidence for thinking that a linearization theorem should be true for actions of semi-simple Lie groups preserving a rigid structure. 
There are many (but non-obvious) ways to show that conformal structures are rigid at order 2 (i.e. of finite type 2 ). In general, one associates to the conformal structure a natural pseudo-Riemannian metric on a natural fiber bundle over $M$. Here, we adopt the approach of [6]. There, one considers the bundle $X^{1}(M)$ of 1 -jets of metric in the conformal class, and shows that it is naturally endowed with a pseudo-Riemannian metric, and with a horizontal distribution $H$, for which the projection $\pi: X^{1}(M) \rightarrow M$ is horizontally conformal, that is for any $u \in X^{1}(M), d \pi_{u}: H_{u} \rightarrow T_{\pi(x)} M$ is conformal. Actually, the advantage of this construction (with respect to the others) is that $X^{1}(M)$ is an affine fiber bundle, i.e its fibers are affine spaces. Now, if $G$ is a group acting conformally on $M$ with a fixed point $x_{0}$, then it acts affinely on $X_{x_{0}}^{1}(M)$. In particular, if $G$ is a semi-simple Lie group, thanks to the standard theorems on vanishing of its $H^{1}$-cohomology (with respect to any representation), one infers that $G$ fixes a point $u_{0}$ in $X_{x_{0}}^{1}(M)$. Let $h=\pi \circ \exp _{u_{0}}: H_{u_{0}} \rightarrow M$, where exp is the exponential map of the pseudo-Riemannian metric on $X^{1}(M)$ (defined on a neighbourhood of the 0 section of $\left.T X^{1}(M)\right)$. Then $h$ conjugates the action of $G$ near $x_{0}$ with the linear action of $G$ on $H_{u_{0}}$ (which is the same via $d \pi_{u_{0}}$ as its linear action on $\left.T_{x_{0}} M\right) \cdot \diamond$

\section{References}

[1] U. Bader, A. Nevo, Conformal actions of simple Lie groups on compact pseudoRiemannian manifolds. J. Differential Geom. 60 (2002), 355-387.

[2] C. Cairns, É. Ghys, The local linearization problem for smooth $\mathrm{SL}(n)$-actions. Enseign. Math. (2) 43 (1997) 133-171.

[3] G. D'Ambra, Isometry groups of Lorentz manifolds, Invent. Math. 92 (1988) 555-565.

[4] G. D'Ambra and M. Gromov, Lectures on transformation groups : geometry and dynamics, Surveys in Differential Geometry (Supplement to the Journal of Differential Geometry), 1 (1991) 19-111.

[5] J. Ferrand, The action of conformal transformations on a Riemannian manifold, Math. Ann. 304 (1996) 277-291.

[6] C. Frances, Les structures conformes pseudo-riemanniennes vues comme structures rigides à l'ordre 2. Preprint (2002). Available at http://www.math.u-psud.fr/ frances.

[7] C. Frances, Géométrie et dynamique lorentziennes conformes. Thesis (2002). Available at http://www.math.u-psud.fr/ frances.

[8] M. Gromov, Rigid transformation groups, "Géometrie différentielle ", D. Bernard et Choquet-Bruhat. Ed. Travaux en cours 33. Paris. Hermann (1988).

[9] S. Kobayashi, Transformation groups in differential geometry. Ergebnisse der Mathematik und ihrer Grenzgebiete, Band 70. Springer-Verlag, New York-Heidelberg, 1972.

[10] N. Kowalsky, Noncompact simple automorphism groups of Lorentz manifolds, Ann. Math. 144 (1997) 611-640.

[11] R. S. Kulkarni, U.Pinkall, Conformal geometry. Aspects of Mathematics, E12. Friedr. Vieweg and Sohn, Braunschweig, 1988.

[12] I. Segal, Mathematical cosmology and extragalactic astronomy. Pure and Applied Mathematics, 68. Academic Press [Harcourt Brace Jovanovich, Publishers], New York-London, 1976.

[13] D. Stowe, Stable orbits of differentiable group actions. Trans. Amer. Math. Soc. 277 (1983), 665-684. 
[14] W. Thurston, A generalization of the Reeb stability theorem. Topology 13 (1974), 347352.

[15] _ Three dimensional geometry and topology. 1, Princeton University Press, 1997. Edited by Silvio Levy.

CNRS, UMPA, ENS Lyon, 46, Allée D'Italie, 69364 Lyon Cedex 07, FRANCE

E-mail address: cfrances@umpa.ens-lyon.fr, Zeghib@umpa.ens-lyon.fr $U R L:$ http://www. umpa.ens-lyon.fr 